\title{
A Image analysis System to Detect Skin Diseases
}

\author{
Pravin S. Ambad ${ }^{1}$, A. S. Shirsat ${ }^{2}$ \\ ${ }^{I}$ Dept. of Electronics and Telecommunication, STES's SKNCOE, Vadgaon, Pune, India \\ ${ }^{2}$ Dept. of Electronics and Telecommunication, STES's SKNCOE, Vadgaon, Pune, India
}

\begin{abstract}
Skin diseases rate has been increasing for past few decades. Psoriasis is chronic inflammatory skin disease which affects more thgan $3 \%$ of population. One of the risk factor in skin cancer is unprotected exposer to UV radiation, which causes various skin diseases. For early diagnosis of skin cancer, psoriasis and dermatophytosis and increases chance for cure significantly. Therefore proposed system used for early prevention and detection named a Image analysis system to detects skin diseases. The image analysis technique where user will able to take skin images of different mole type or rashes type. System will process and analyse the images, which provide notification to user you need medical help. This system provides automatic skin diseases prevention and detection.)
\end{abstract}

Keywords: Enhancement, Segmentation, statistical analysis, Adaboost classifier.

\subsection{Background and Motivation}

\section{Introduction}

Now a day's people are suffering from skin diseases, More than 125 million people suffering from Psoriasis also skin cancer rate is rapidly increasing over last few decades specially Melanoma is most diversifying skin cancer. Dermatophytosis rate is high specially at rural areas.

If skin diseases are not treated at earlier stage, then it may lead to complications in the body including spreading of the infection from one individual to the other. The skin diseases can be prevented by investigating the infected region at an early stage. The characteristic of the skin images are diversified, so that it is challenging job to devise an efficient and robust algorithm for automatic detection of the skin disease and its severity. Skin tone and skin color plays an important role in skin disease detection. Colour and coarseness of skin are visually different. Automatic processing of such images for skin analysis requires quantitative discriminator to differentiate the diseases.

Proposed system is combo model which is used for the prevention and early detection of skin cancer, psoriasis and Dermatophytosis. Basically skin disease diagnosis depends on the different characteristics like color, shape, texture etc. there are no accepted treatment for skin diseases Different physicians will treat differently for same symptoms. Key factor in skin diseases treatment is early detection further treatment reliable on the early detection.

In this paper, Proposed system is used for the diagnosis multiple skin disease using statistical parameter analysis. Statistical analysis is anxious with analysis of random data. Random data is pattern of skin diseases. Standard database is used this data does not have any mathematical expression, it has some statistical properties. To analyses random data we must analyze statistical properties of it.

\subsection{Contribution}

In this paper, we present Image analysis system to diagnosis multiple skin disease using statistical parameter analysis. Statistical analysis is concerned with analysis of random data. This system is combo-model which is to be used to diagnosis multiple skin diseases at a time. The target skin diseases are skin cancer, psoriasis and dermatophytosis. The disease diagnosis and classification is built on statistical parameter analysis. Statistical parameters includes: Entropy, Texture index, Standard deviation, Correlation fact Depending on standard range of parameters skin disease is going to be diagnosis and classified.

\subsection{Paper Organization}

Paper is ordered as follows: Section II related work. Section III proposed system named image analysis system to detection of skin diseases. Section IV describes the technique for skin disease images classification. In Section V It shows the results of the classification framework. In Section VI It conclude the paper with future work 


\section{Relegated Work}

Skin image identification has become one of the most demanding and attractive research areas in the past few years. Colour histogram based characteristics are used to analyse and classify the psoriasis infected skin images in sequence to take the diagnostic measures [3]. On one hand, this would be useful for dermatologists to decrease diagnostic faults, while on the other hand it can help as the early test bed for patients in rural areas where there is a dearth of good medical professionals. A support Vector Machine with RBF kernel is used for the classification of images. The experimental results gave the encouraging results in an initial attempt for identification of psoriasis infected skin images. Classification of psoriasis skin diseases and their severity will be carried out.

Image processing method is implemented in MATLAB, for skin cancer detection. In this paper, online database of skin cancer images is used for testing the method [4]. Skin images for cancers of different types are obtained from, of these images for BCC (Basal cell carcinoma), SCC (squamous cell carcinoma) and normal or harmless skin lesions are collected and database is created for testing purpose Next step in image processing is de-noising using wavelet tool. To remove low frequency or background noise from image, filtering is used. After de-noising the image, median filtering is applied to remove some hair like material from skin image, if present Thresholding is the simplest and most commonly used method of segmentation. This paper considers use of high-level feature extraction technique by implementing the 2D-DWT as the processing method neural network tool is used for classification of skin images in different skin diseases. Using the features extracted from image, it is classified either in harmful diseased i.e., BCC (Basel cell carcinoma), SCC (Squamous cell carcinoma), or harmless.

Buket D. have worked on real time system for the malignant melanoma prevention and early detection [5]. In this system user is able to analyse captured image. System process the image and shows the notification for medical help. This system shows convincing results and accuracy.

Ho Tak Lau. Have worked on an automatic skin cancer classification [6]. Available image is given to the system and it goes through different image processing procedure. Use full information is extracted from the image and then with the help of training and testing system classifies the image. Recognition accuracy of the neural network classifier is $90 \%$
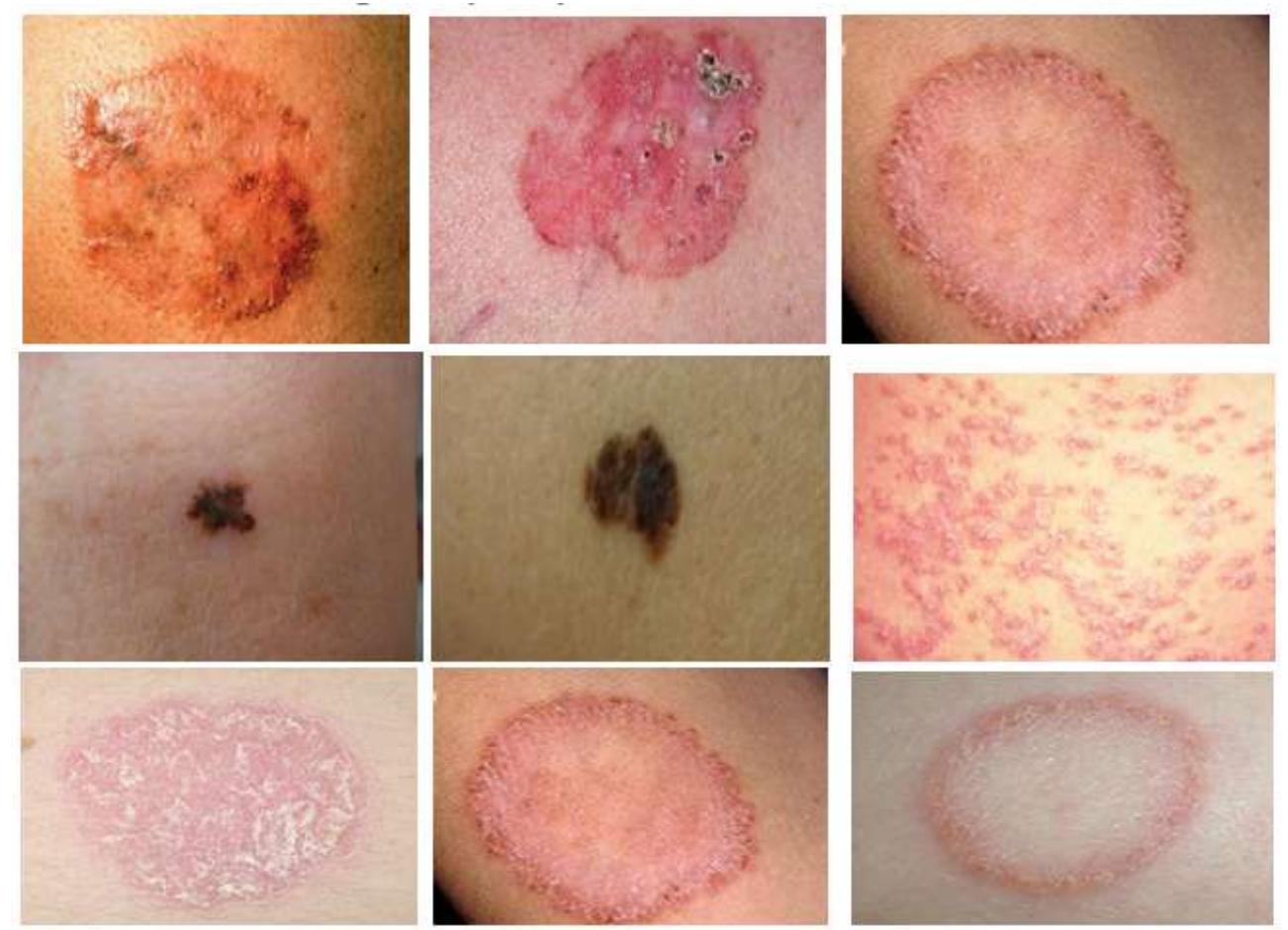

Fig 1. Sample images from database including melanom, psoriasis and dermatophytosis.

\section{Proposed System}

In this paper we propose the Image analysis system to detect skin diseases. Our system capture image from standard database and put in to the system to inform the user for preventing the threats linked to skin diseases. More briefly, we present the Image analysis system to detect different skin diseases, where user will able to take images of different moles or skin patches. Our system will analyze and process the image and classifies the image to normal, melanoma, psoriasis or dermo case based extracting the image features. An alert 
will be provided to the user to seek medical help if the mole belongs to the atypical or melanoma category. Major components in proposed system flow chart shown in fig2 and Architecture of system is shown in fig 3.

This database includes images of multiple skin diseases. These images are occupied from standard database shown in fig 1 . The image database contains a total of 130 images of lesions, including melanoma, psoriasis and dermatophytosis cases.

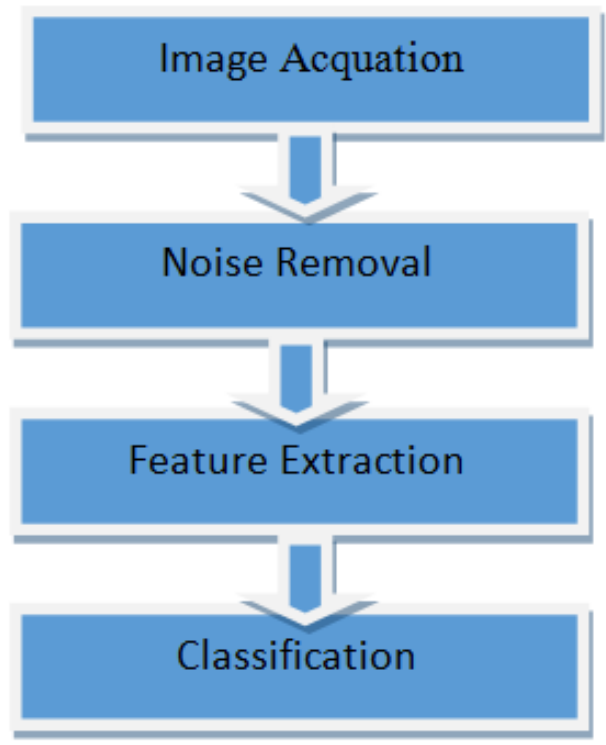

Fig 2. Flow Chart of Proposed Method.

- Image Acquation

- Noise Removal

- Segmentation

- Feature Extraction

- Classification

\subsection{Image Acquation}

The first stage of our automated image analysis system is image acquisition. This stage is essential for the rest of the system; hence, if the image is not acquired satisfactorily, then the remaining components of the system may not be achievable, or the results will not be reasonable. In this stage first image sytem requires the resized image for the better results. Input image given to the system is in RGB form. But for our proposed system requires gray images. Hence using rgb to gray conversion in MATLAB we convert RGB images in to gray images.

\subsection{Noise Removal}

It's necessary to have quality images without any noise to get accurate result. Noisy image may lead your algorithm towards incorrect result. Hence it becomes necessary to de-noise the image. Image de noising is an important image processing task, There are many ways to de noise an image. The important for good image de noising model is that it will remove noise while preserving edges. Traditionally, linear models have been used. To de-noise the image we can use median filter. Median filter does the work of smoothening of image.

\subsection{Feature Extraction}

To get accurate result in biomedical image processing it is always necessary that biomedical image must be of very good quality. However, practically this is not easy. Due to different reasons obtain low or medium quality images. Hence it becomes necessary to improve their quality. To improve the quality of image using image enhancement algorithm. This algorithm enhances the image by focusing on parameters like contrast, brightness adjustment

\subsubsection{Statistical Analysis:}

Statistical analysis of skin image is supposed to give different statistics such as: Entropy Standard deviation Texture factor correlation factor Depending on this parameter first find out there range for individual skin disease. To find out range of these parameters used for standard skin disease image database.

- We calculate the mean 


$$
m_{I}=\frac{\sum_{i=11}^{12} I_{i}(i)}{l 2-l 1}
$$

- First we calculate the variance with the benefit of sample mean

$$
\sigma_{I}^{2}=\frac{\sum_{i=11}^{\prod_{11}}\left(l_{1}(i)-m_{\mathrm{i}}\right)^{2}}{l_{2}-l_{1}}
$$

- With the benefit of variance, we calculate the energy of the image I by taking mean square of variance.

- We then calculate the luminance of image with the benefit of RGB components of image I, we calculate the RGB components of image by taking mean of R,G and B components.

$$
\begin{aligned}
R_{n} & =\frac{R}{R+G+B} \\
G_{n} & =\frac{G}{R+G+B} \\
B_{n} & =\frac{B}{R+G+B} \\
L & =\sqrt{\left(\left(0.3 * r^{2}\right)+\left(0.587 * g^{2}\right)+\left(0.114 * b^{2}\right)\right)}
\end{aligned}
$$

- We calculate sample similarity measurement with the benefit of resized image $\mathrm{I} 2$, texture $\mathrm{T} 2$, window and luminance $\mathrm{L}$.

- We calculate the standard deviation of image

$$
\sigma_{\mathrm{i}}=\sqrt{\sigma_{\mathrm{i}}^{2}}
$$

- We calculate the entropy of the image

$$
\text { entropy }=-\operatorname{sum}\left(p \cdot * \log ^{2}(\mathrm{p})\right) \text {. }
$$

\subsection{Classification}

In this frame two-level classifiers. At first level it classifies normal or abnormal and second level it classifies in specified category i.e Melanoma, Psoriasis or dermo The first stage of this framework is to perform image processing to detect and exclude the noise, after that the ROI of the skin lesion is segmented. Then, the image features are extracted. Next, the extracted features are fed to the classifier

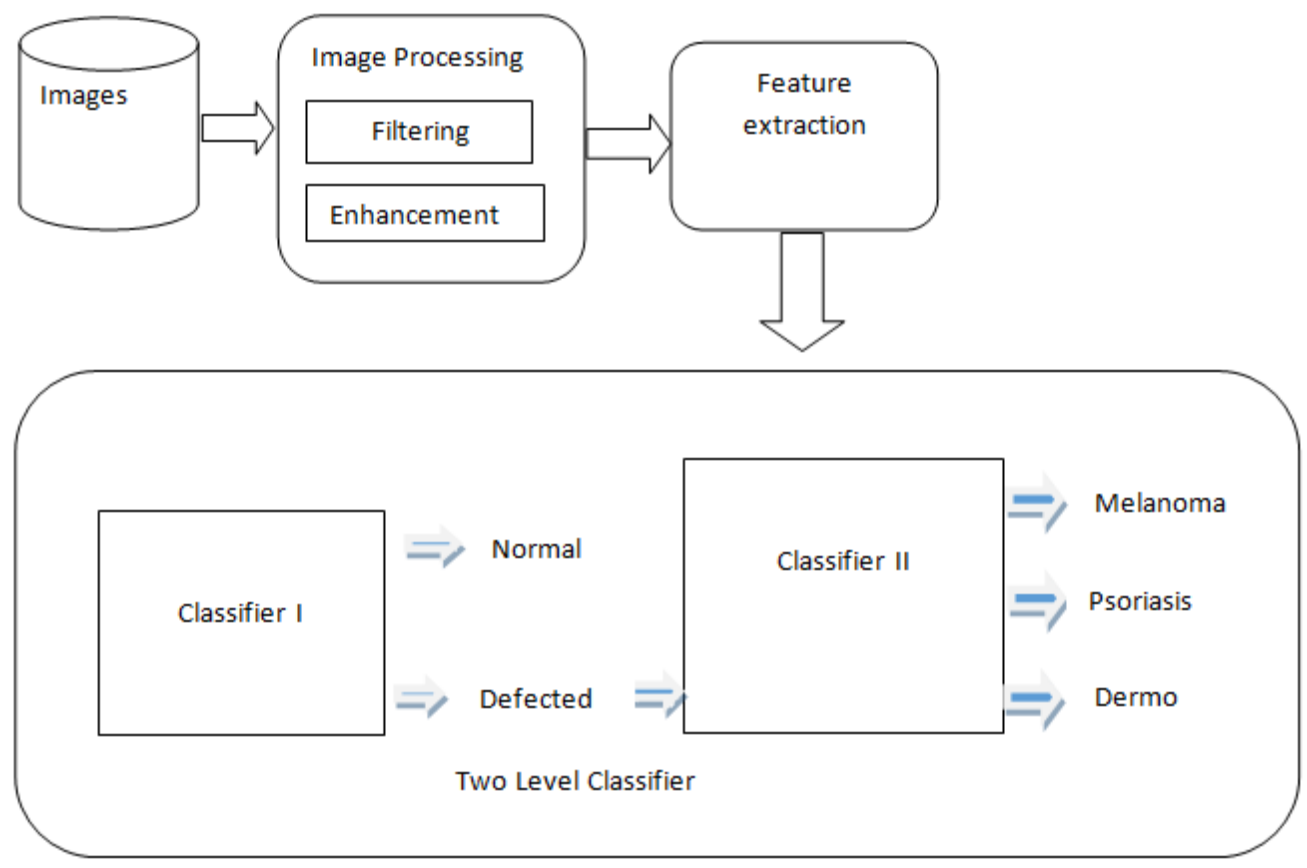

Fig.3. Architecture of proposed method 


\section{Result Simulation}

In proposed system, we use standard database for the development and testing of proposed system. A framework Proposed system is shown in fig 3. At first stage this framework performs image processing for denoise the image and enhancement the image for statistical analysis. Shown in fig 4. After filtering feature extraction is applied, It calculate the entropy, Standard deviation, texture factor to discover the range of parameters used in image. Table 1 shows the range of statistical parameters of images as per there category. We use two leval classifier to get better results. With the benefit of AdaBoost classifier it correlate the images with deciding the range of correlation by the assistance of mean, standard deviation based on intensity classifier classifies biomedical images.

Input Image
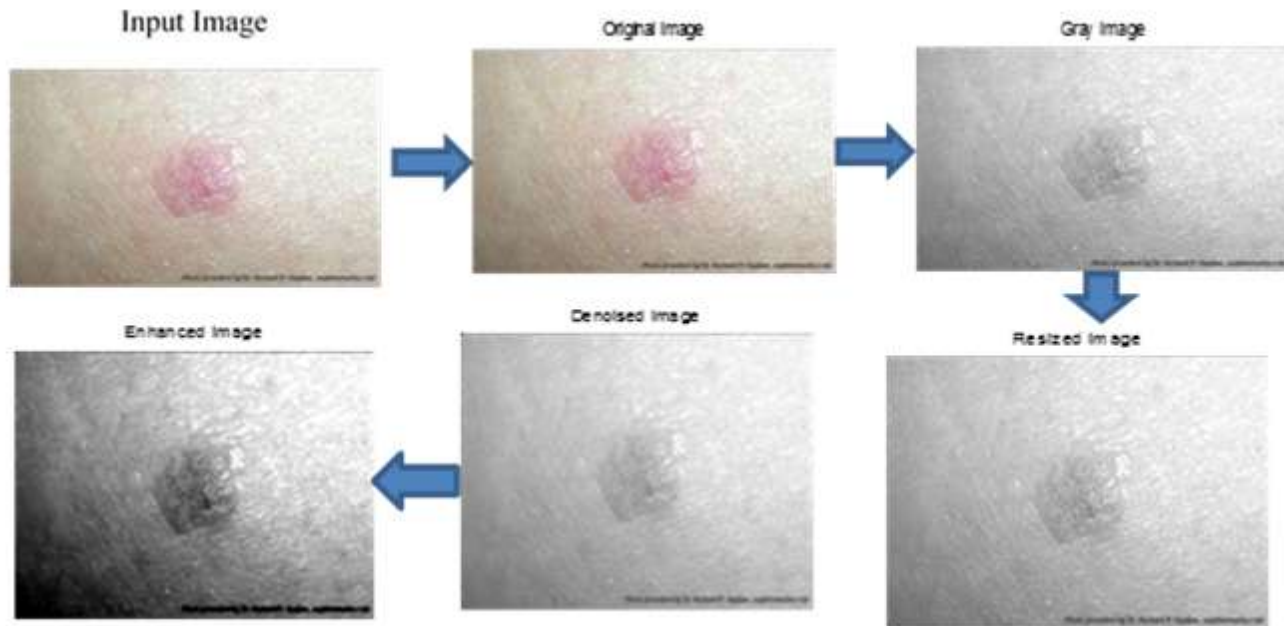

Denolses ims $x$
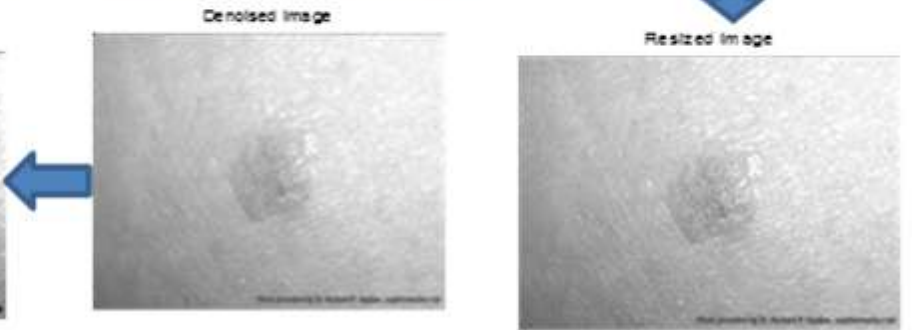

Fig 4. Input image, Filtered image, enhanced image.

Table 1.Results for statistical parameter analysis

\begin{tabular}{|l|l|l|l|l|}
\hline & Luminance & Texture & Standard Deviation & Entropy \\
\hline Psoriasis & $200.1013-225.3665$ & $0.1853-0.2404$ & $9.5709-30.91$ & $5.065-6.7293$ \\
\hline Melanoma & $192.0704-100.2521$ & $0.2764-0.1645$ & $60.6499-21.1382$ & $7.4575-5.5998$ \\
\hline Dermo & $151.4497-196.3330$ & $0.2011-0.2733$ & $11.0351-54.5073$ & $5.4555-7.5061$ \\
\hline Normal skin & $226.8690-244.7091$ & $0.2235-0.2400$ & $4.0219-19.6765$ & $3.6991-5.6361$ \\
\hline
\end{tabular}

In the proposed framework as result of adaboost classifier, we can classify the normal, psoriasis, dermo and melanoma images with accuracy of $90 \%$ or more. Table II shows results for standard deviation. This automated image analysis module where Image processing module classifies under which category the image falls (Psoriasis, Melanoma and Dermatophytosis).

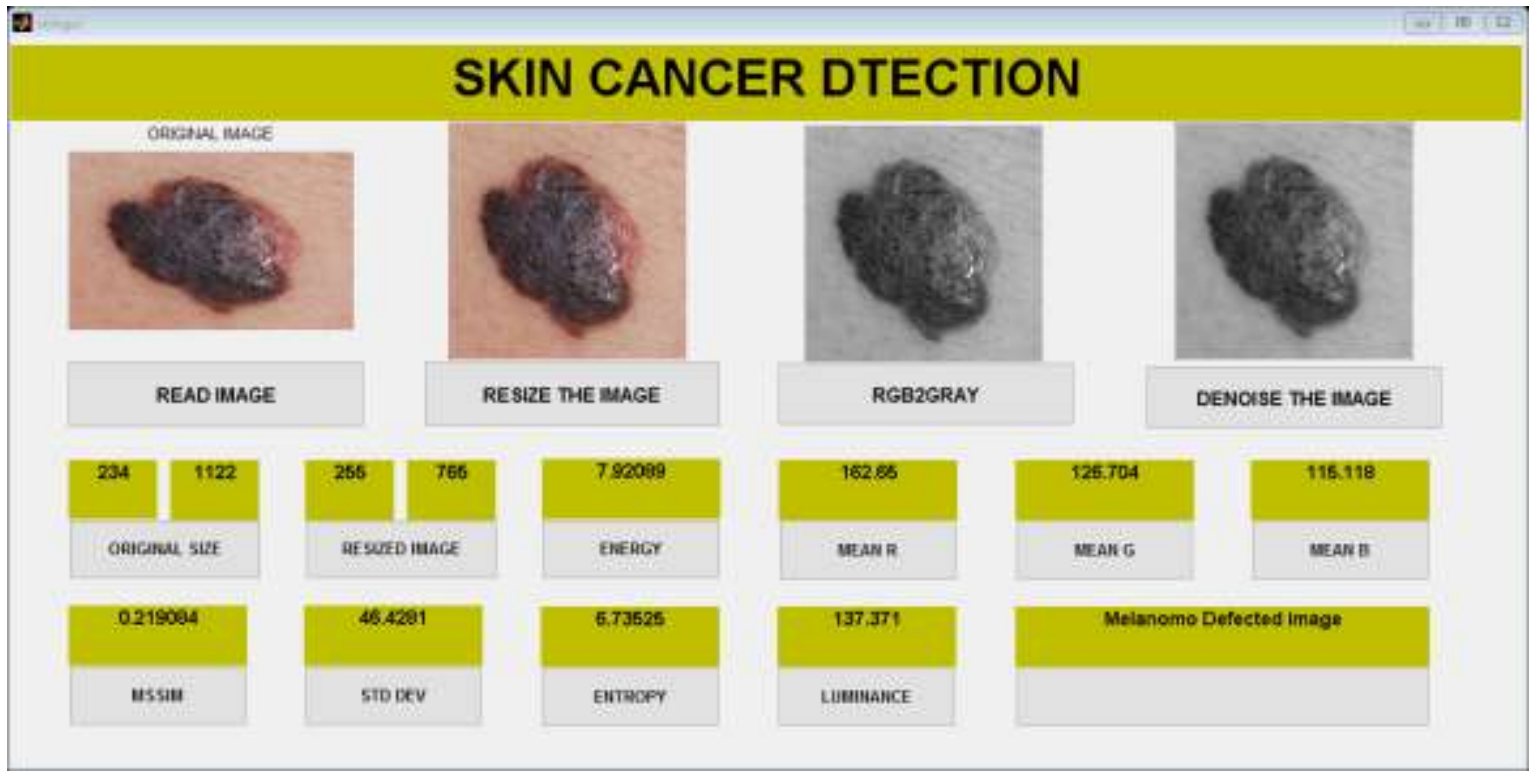

Fig 5. Result for melanboma. 


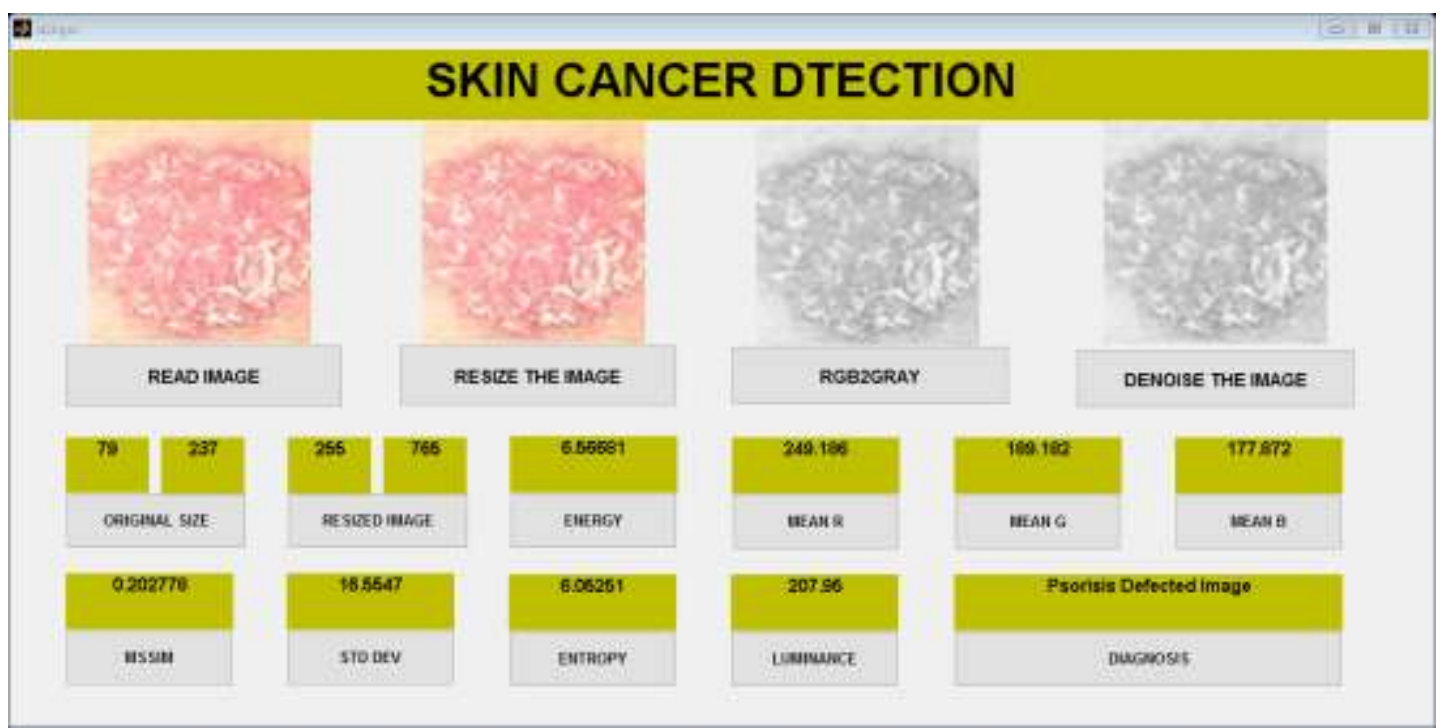

Fig 6. Result for psoriasis

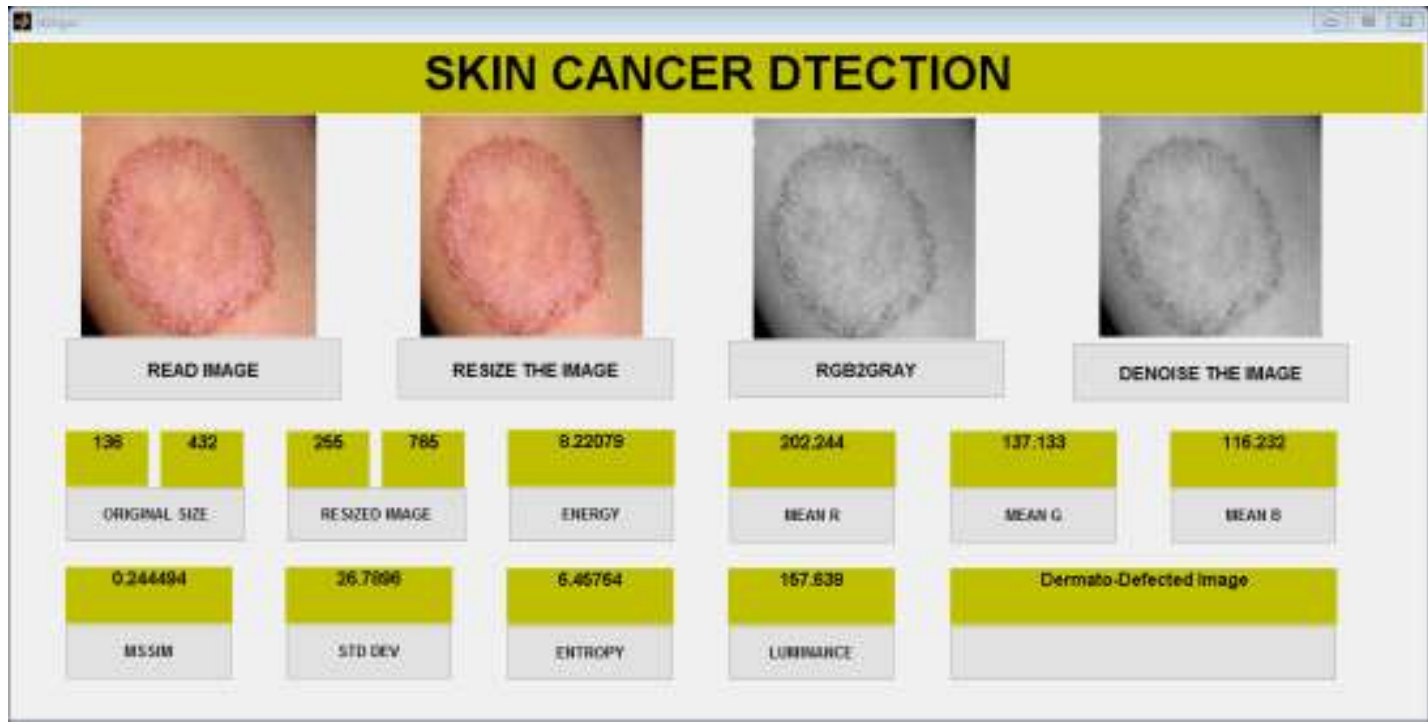

Fig 7. Results for dermo.

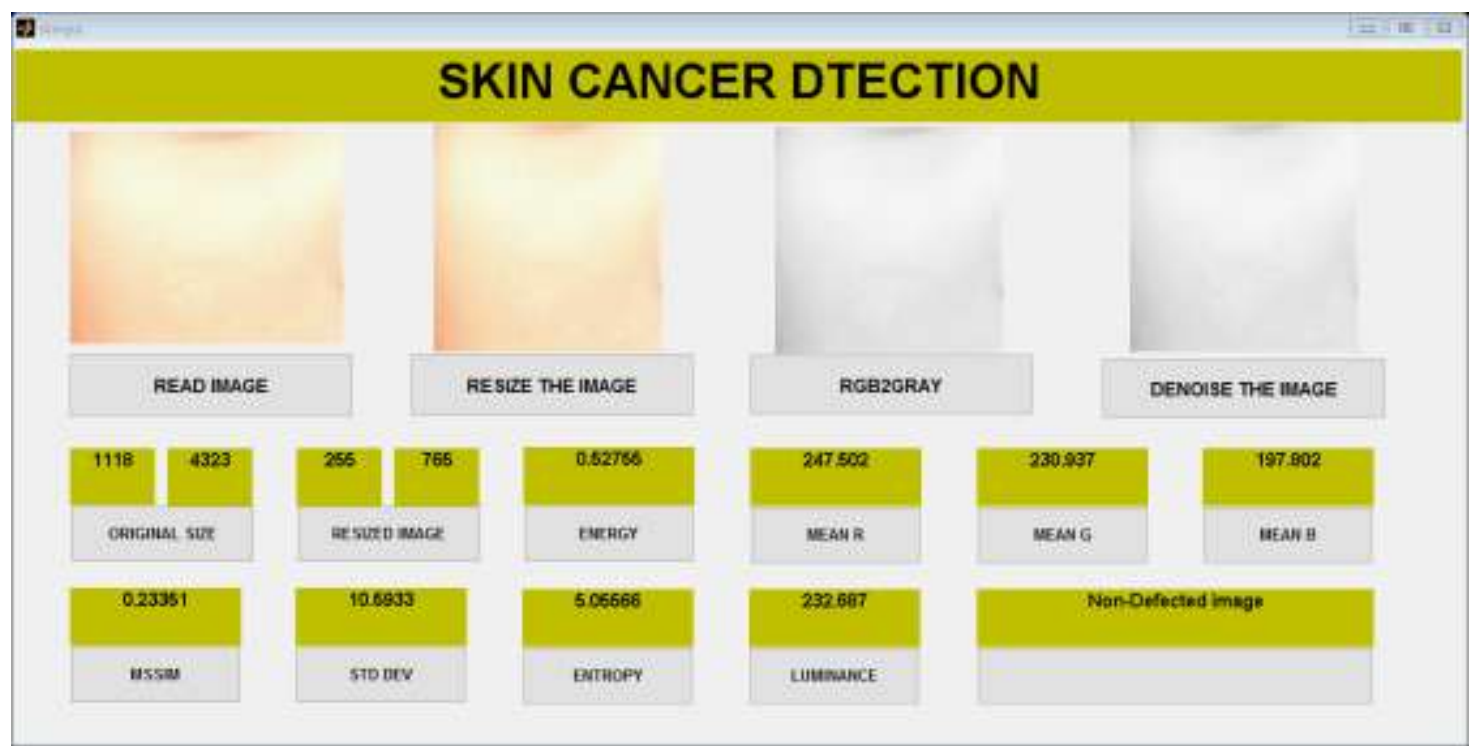

Fig 8. Result for normal skin. 


\section{Conclusion}

In this paper we present Image analysis system for prevention and detection of skin diseases. Using statistical analysis with correlation algorithm we can diagnosis the multiple skin diseases as well as classify skin disease. Different statistical parameters has been studied amongst them are Entropy, Texture index, Correlation factor has been chosen to find out probability of disease. According to further requirement if necessary statistical parameters can be increased. Outcome of this system is supposed to diagnosis the multiple skin diseases as well as it classifies skin diseases. This future work may reduce the processing time of diffusion speed. That makes system faster.

\section{Acknowledgements}

It is my7 pleasure to get this opportunity to thank my beloved and respected guide Prof A. S. Shirsat who improved valuable basic knowledge of Electronics and Telecommunication specially related to Signal Processing domain. We are greateful to Electronics and Telecommunication department of SMTs SKNCOE, vadgaon (Bk), pune for providing infrastructure facilities and more support.

\section{References}

[1]. B.V.Dhandra, Shridevi Soma, Shweta Reddy, "Color Histogram Approach For Analysis Of Psoriasis Skin Disease", IEEE system Journal, vol.99,pp. 25-29.

[2]. A. Karargyris, O. Karargyris, A. Pantelopoulos, "DERMA/ care: An Advanced Image- Processing Mobile Application for Monitoring Skin Cancer,"'in IEEE $24^{\text {th }}$ International Conference on Tools with Artificial intelligence(ICTAI), 2012, PP.1-7

[3]. R. Siegel, D. Naishadhama, A. Jemal, “Cancer Statistics, 2012,”CA: a cancer journal for clinicians, vol.62,2012, pp.10-29.

[4]. T. Wadhawan, N. Situ, K. Lancaster, X. Yuan, G. Zouridakis, "SkinScan: A Portable Library for Melanoma Detection On Handheld devicees," in IEEE International Symposium On Biomedical Images: from nano to micro,2011,2011,pp.133-136.

[5]. Omar Abuzaghleh, Buket D. Barkana, Miad Faezipour, "SKIN cure: A Real Time Image Analysis System to Aid in the malignant melanoma Prevention and Early Detection."Member IEEE SSIAI 2014, pp. 85-88.

[6]. Ho Tak Lau, Adel Al-Jumaily, "Automatically early detection of skin cancer," International conference of soft computing and pattern recognition, IEEE 2009, pp.375-380.

[7]. Romero G, García M, Vera E, Martínez C, Cortina P, Sánchez P, Guerra A. "Preliminary results of DERMATEL: prospective randomized study comparing synchronous and asynchronous modalities of teledermatology." Actas Dermosifiliogr. 2006 Dec; vol.97 (10): pp 630-36.

[8]. Scherr, D.; Zweiker, R.; Kollmann, A.; Kastner, P.; Schreier, G. \& Fruhwald, F. "Mobile phone based surveillance of cardiac patients at home Journal of Telemedicine and Telecare," 2006,vol 5, pp 255-261.

[9]. Anthony Teik Jin Goon, Gil Yosipovitch, Yiong Huawk Chan and Chee Leok Goh,"Barrier repair in chronic plaque type Psoriasis." Skin and Research Technology 2004, vol. 7, pp1-7.

Table II. Parameters for the melanoma diseases images

\begin{tabular}{|c|c|c|c|c|c|}
\hline Melanoma Image name & Energy & Luminance & Texture & Standard deviation & Entropy \\
\hline 1. & 2.1395 & 111.3409 & 0.2884 & 18.3976 & 5.8905 \\
\hline 2. & 3.3612 & 100.2521 & 0.2764 & 21.1382 & 6.2428 \\
\hline 3. & 6.2569 & 107.0817 & 0.2218 & 59.3111 & 6.7048 \\
\hline 4. & 4.6866 & 131.6434 & 0.2829 & 0.2829 & 6.8299 \\
\hline 5. & 9.1803 & 184.2448 & 0.2048 & 60.6499 & 6.4348 \\
\hline 6. & 14.7339 & 102.3955 & 0.1847 & 45.8963 & 7.4555 \\
\hline 7. & 7.8489 & 157.9357 & 0.2263 & 38.7305 & 6.9676 \\
\hline 8. & 6.2232 & 192.0704 & 0.2238 & 34.1982 & 6.1984 \\
\hline 9. & 17.5618 & 179.1952 & 0.2109 & 54.2302 & 6.5156 \\
\hline 10. & 7.8602 & 132.7504 & 0.2477 & 30.2290 & 6.8447 \\
\hline 11. & 6.1686 & 159.4787 & 0.2350 & 46.7928 & 7.2443 \\
\hline 12. & 4.1057 & 185.9057 & 0.2354 & 28.4909 & 5.5998 \\
\hline 13. & 16.3758 & 132.9732 & 0.2216 & 56.0052 & 7.4575 \\
\hline 14. & 11.7062 & 119.5764 & 0.1799 & 55.7795 & 7.3596 \\
\hline 15. & 16.6170 & 132.4336 & 0.2210 & 56.1507 & 7.4417 \\
\hline 16. & 7.1511 & 137.7104 & 0.2390 & 14.1188 & 5.8422 \\
\hline 17. & 18.6874 & 174.3030 & 0.1645 & 31.9370 & 6.8998 \\
\hline 18. & 6.1526 & 169.4482 & 0.2355 & 29.7860 & 6.2005 \\
\hline 19. & 4.1152 & 149.2433 & 0.2455 & 40.4684 & 6.3816 \\
\hline 20. & 7.9209 & 137.3714 & 0.2191 & 46.4281 & 6.7352 \\
\hline 21. & 21.3086 & 182.6758 & 0.2307 & 52.2923 & 5.6645 \\
\hline 22. & 8.6039 & 154.2731 & 0.2547 & 38.6597 & 6.6992 \\
\hline 23. & 9.4501 & 117.4625 & 0.2291 & 35.2706 & 7.0187 \\
\hline 24. & 7.2039 & 165.0435 & 0.2435 & 33.9218 & 6.7276 \\
\hline 25. & 5.3904 & 168.9671 & 0.2257 & 19.1702 & 5.6177 \\
\hline 26. & 7.3446 & 132.4633 & 0.2153 & 46.5743 & 7.3636 \\
\hline 27. & 3.4853 & 145.6335 & 0.2523 & 33.6139 & 6.6680 \\
\hline
\end{tabular}


A Image analysis System to Detect Skin Diseases

\begin{tabular}{|c|l|l|l|l|c|}
\hline 28. & 3.8173 & 128.6231 & 0.2480 & 32.4758 & 6.4711 \\
\hline 29. & 4.0282 & 179.5976 & 0.2380 & 24.8413 & 6.3430 \\
\hline 30. & 4.5983 & 154.9212 & 0.2302 & 34.7408 & 6.6368 \\
\hline 31. & 4.3651 & 164.3595 & 0.2618 & 26.8596 & 6.6779 \\
\hline 32. & 2.3708 & 125.1829 & 0.2707 & 22.9876 & 5.9407 \\
\hline Max- $\min$ & $18.6874,2.3708$ & $\mathbf{1 9 2 . 0 7 0 4}$, & $0.2764, \mathbf{0 . 1 6 4 5}$ & $\mathbf{6 0 . 6 4 9 9 , 2 1 . 1 3 8 2}$ & $\mathbf{7 . 4 5 7 5 , 5 . 5 9 9 8}$ \\
& & $\mathbf{1 0 0 . 2 5 2 1}$ & & & \\
\hline
\end{tabular}

Table III. Parameters for the Psoriasis diseases images

\begin{tabular}{|c|c|c|c|c|c|}
\hline Psoriasis skin images & energy & luminance & texture & Standard deviation & entropy \\
\hline 1 & 4.3180 & 222.1059 & 0.2066 & 16.7222 & 6.0351 \\
\hline 2 & 5.7618 & 205.7455 & 0.2170 & 22.8729 & 6.4621 \\
\hline 3 & 1.9985 & 224.4457 & 0.2305 & 13.8666 & 5.7231 \\
\hline 4 & 1.6342 & 200.0955 & 0.2381 & 11.4235 & 5.3957 \\
\hline 5 & 6.5668 & 207.9505 & 0.2028 & 16.5547 & 6.0525 \\
\hline 6 & 5.6464 & 211.7912 & 0.2243 & 27.7675 & 6.6865 \\
\hline 7 & 4.0449 & 207.6458 & 0.2321 & 20.0038 & 6.2242 \\
\hline 8 & 3.9039 & 225.0790 & 0.2217 & 25.1186 & 6.2018 \\
\hline 9 & 2.2044 & 222.7065 & 0.2265 & 10.8031 & 5.4017 \\
\hline 10 & 1.0527 & 208.6775 & 0.2385 & 8.4837 & 4.8725 \\
\hline 11 & 4.0110 & 224.6513 & 0.2100 & 17.3597 & 5.9935 \\
\hline 12. & 2.8139 & 210.1426 & 0.2310 & 14.4597 & 5.7885 \\
\hline 13 & 5.4650 & 208.7148 & 0.2109 & 20.3990 & 6.2245 \\
\hline 14 & 3.0569 & 216.3364 & 0.2172 & 12.9045 & 5.6879 \\
\hline 15 & 2.9442 & 207.0539 & 0.2293 & 11.2046 & 5.4196 \\
\hline 16 & 4.0749 & 200.1013 & 0.2250 & 10.8009 & 5.4465 \\
\hline 17 & 6.2680 & 220.5977 & 0.1853 & 20.0102 & 6.0656 \\
\hline 18 & 5.4808 & 206.2831 & 0.2051 & 14.0411 & 5.8280 \\
\hline 19 & 1.3932 & 213.2058 & 0.2404 & 12.1803 & 5.5225 \\
\hline 20 & 2.7538 & 220.6839 & 0.2293 & 19.4234 & 6.2329 \\
\hline 21 & 3.5546 & 225.3665 & 0.2194 & 24.7035 & 6.1190 \\
\hline 22 & 3.4873 & 215.3503 & 0.2203 & 13.9779 & 5.7515 \\
\hline 23 & 4.9963 & 216.7179 & 0.2183 & 25.3859 & 6.4872 \\
\hline 24 & 2.3789 & 215.2518 & 0.2209 & 12.5248 & 5.6085 \\
\hline 25 & 2.9326 & 208.0418 & 0.2380 & 18.1444 & 6.1597 \\
\hline 26 & 2.9199 & 214.3392 & 0.2295 & 21.6772 & 6.2863 \\
\hline 27 & 4.2087 & 212.0241 & 0.2325 & 30.9100 & 6.7293 \\
\hline 28 & 8.7774 & 212.7213 & 0.1861 & 23.0166 & 6.4354 \\
\hline 29 & 2.9797 & 206.1947 & 0.2203 & 8.8792 & 5.1489 \\
\hline \multirow[t]{2}{*}{30} & 4.6783 & 218.9145 & 0.2076 & 13.6307 & 5.7690 \\
\hline & $1.0527-8.7774$ & $200.0955-225.3665$ & $0.1853-0.2404$ & 9.5709- 30.91 & $5.065-6.7293$ \\
\hline
\end{tabular}

Table IV. Parameters for the dermo diseases images

\begin{tabular}{|c|c|c|c|c|c|}
\hline Dermo skin images & energy & luminance & texture & Standard deviation & entropy \\
\hline 1. & 8.2208 & 157.6380 & 0.2445 & 26.7896 & 6.4576 \\
\hline 2. & 2.9190 & 171.0626 & 0.2534 & 21.7858 & 6.3542 \\
\hline \multicolumn{6}{|l|}{3.} \\
\hline 4. & 13.2996 & 169.0525 & 0.2436 & 48.9335 & 7.5061 \\
\hline 5. & 9.2580 & 167.4760 & 0.2151 & 31.6206 & 6.8984 \\
\hline 6. & 0.9160 & 170.3948 & 0.2641 & 11.0351 & 5.4555 \\
\hline 7. & 3.2053 & 196.3330 & 0.2305 & 21.0784 & 6.2085 \\
\hline 8. & 3.8366 & 191.7152 & 0.2357 & 18.9532 & 6.2202 \\
\hline \multicolumn{6}{|l|}{9.} \\
\hline 10. & 6.8552 & 179.0907 & 0.2645 & 33.6863 & 6.7403 \\
\hline 11. & 26.1018 & 170.6602 & 0.2011 & 35.5133 & 6.8550 \\
\hline 12. & 2.7319 & 184.9130 & 0.2508 & 25.3052 & 6.3360 \\
\hline 13. & 5.3201 & 170.0740 & 0.2320 & 27.4864 & 6.5279 \\
\hline 14. & 4.3678 & 156.5844 & 0.2434 & 20.2846 & 6.2979 \\
\hline 15. & 4.3509 & 192.0466 & 0.2351 & 32.0045 & 6.8368 \\
\hline 16. & 3.2639 & 179.2251 & 0.2423 & 18.0502 & 6.0254 \\
\hline 17. & 3.8865 & 158.6846 & 0.2733 & 23.8142 & 6.4957 \\
\hline 18. & 26.1018 & 170.6602 & 0.2011 & 35.5133 & 6.8550 \\
\hline 19. & 2.4850 & 167.9784 & 0.2516 & 19.1181 & 6.1942 \\
\hline \multicolumn{6}{|l|}{20.} \\
\hline 21. & 8.3585 & 151.4497 & 0.2469 & 41.2455 & 7.1662 \\
\hline 22. & 5.0942 & 191.5825 & 0.2328 & 26.7727 & 6.7303 \\
\hline 23. & 4.3486 & 186.3283 & 0.2565 & 26.4227 & 6.6447 \\
\hline 24. & 7.0730 & 157.5391 & 0.2389 & 24.8450 & 6.6186 \\
\hline 25. & 11.8521 & 169.0297 & 0.2318 & 54.5073 & 7.4751 \\
\hline Max- Min & $0.9160-26.1018$ & $151.4497-196.3330$ & $0.2011-0.2733$ & $11.0351-54.5073$ & $5.4555-7.5061$ \\
\hline
\end{tabular}


A Image analysis System to Detect Skin Diseases

Table V. Parameters for the normal skin images

\begin{tabular}{|l|l|l|l|l|l|}
\hline Normal skin images & Energy & \multicolumn{1}{|c|}{ luminance } & \multicolumn{1}{c|}{ texture } & Standard deviation & entropy \\
\hline $\mathrm{n} 1$ & 1.6898 & 236.8670 & 0.2328 & 19.6765 & 5.5400 \\
\hline $\mathrm{n} 2$ & 0.5275 & 232.6871 & 0.2335 & 10.5933 & 5.0557 \\
\hline $\mathrm{n} 3$ & 1.6763 & 228.2365 & 0.2364 & 19.4857 & 5.5212 \\
\hline $\mathrm{n} 4$ & 0.1647 & 236.0787 & 0.2320 & 4.0219 & 3.6991 \\
\hline $\mathrm{n} 5$ & 232.2688 & 0.2321 & 8.0032 & 4.8052 \\
\hline $\mathrm{n} 6$ & 0.6509 & 226.8690 & 0.2400 & 14.1614 & 5.6361 \\
\hline $\mathrm{N} 7$ & 1.1770 & 232.8777 & 0.2335 & 5.0142 & 4.2139 \\
\hline $\mathrm{n} 8$ & 0.1610 & 244.7091 & 0.2235 & 6.1332 & 4.4416 \\
\hline $\mathrm{n} 9$ & 0.1579 & 232.1566 & 0.2341 & 8.2052 & 4.9067 \\
\hline $\mathrm{n} 10$ & 0.3541 & 233.3500 & 0.2319 & 11.6723 & 5.1239 \\
\hline & 0.5686 & $226.8690-244.7091$ & $0.2235-0.2400$ & $4.0219-19.6765$ & $3.6991-5.6361$ \\
\hline
\end{tabular}

